\title{
Synthesis of a New Polycarboxylate at Room Temperature and Its Influence on the Properties of Cement Pastes with Different Supplementary Cementitious Materials
}

\author{
Shuncheng Xiang $\mathbb{D}^{1}{ }^{1}$ Yingli Gao, ${ }^{1}$ and Caijun $\mathrm{Shi}^{2}$ \\ ${ }^{1}$ Key Laboratory of Special Environment Road Engineering of Hunan Province, Changsha University of Science \& Technology, \\ Changsha 410114, Hunan, China \\ ${ }^{2}$ College of Civil Engineering, Hunan University, Changsha 410082, China
}

Correspondence should be addressed to Shuncheng Xiang; 410571462@qq.com

Received 16 March 2020; Accepted 29 May 2020; Published 16 June 2020

Academic Editor: Xue Luo

Copyright (c) 2020 Shuncheng Xiang et al. This is an open access article distributed under the Creative Commons Attribution License, which permits unrestricted use, distribution, and reproduction in any medium, provided the original work is properly cited.

\begin{abstract}
Three polycarboxylates with different comb structures (i.e., the same degree of polymerization in side chains but different main chains) were synthesized via radical polymerization reaction at room temperature. The effect of polycarboxylates on the surface tension and the flowability in cement pastes was determined. The best product was selected to study its effects on the hydration heat evolution, compressive strength, autogenous shrinkage, and drying shrinkage of cement pastes with different kinds and contents of supplementary cementitious materials. The results showed that with the increase of molar ratio between AA and TPEG to $6: 1$, we could synthesis the best product. When the water-binder ratio was 0.4 , with the increase of polycarboxylates, the cement hydration heat evolution had been slowed down, and the more the dosage was, the more obvious the effect was. Adding supplementary cementitious materials to cement under the same experimental conditions also played a mitigation role in slowing down the hydration heat. When the water-binder ratio was 0.3 , supplementary cementitious materials could increase the strength of cement by $24.5 \%$ in maximum; its autogenous shrinkage and drying shrinkage could be decreased, respectively, by $60.1 \%$ and $21.9 \%$ in the lowest.
\end{abstract}

\section{Introduction}

Polycarboxylate contained many functional groups including $-\mathrm{COOH},-\mathrm{SO}_{3} \mathrm{H},-\mathrm{NH}_{2},-\mathrm{OH}$, and other groups. These groups could be adsorbed on the hydration products of cement and its surface particles. Not only the adsorption layer was formed, but it could also damage the flocculation of silicate particles. After incorporation of polycarboxylate, its electrostatic repulsion and steric hindrance could significantly change the role of force and solid particles of cement-liquid interface of the physical and chemical properties, and consequently, the particle distribution of cement, thereby affecting the flowability and other properties of cement pastes [1-3]. Currently, however, synthesis of polycarboxylate was commonly used in the heating method. The temperature was between 60 and $90^{\circ} \mathrm{C}$ or even higher. The product had relatively low solid content, so that in the synthesis and the transport process, the cost was increased inadvertently. Thus synthesizing polycarboxylate at room temperature had become a trend in the development of the industry.

Supplementary cementitious materials (SCMs) had become an essential component in the process of preparation of concrete nowadays, including slag, fly ash, silica fume, metakaolin, and other commonly used materials. Their effect on the performance of concrete was significant [4] including hydration heat evolution, compressive strength, autogenous shrinkage, and drying shrinkage.

In this paper, several different polycarboxylates with comb structure were synthesized and selected to study their effects on the properties of cement pastes with different supplementary cementitious materials to provide some theoretical basis for structural design, synthesis, and selection of polycarboxylate. 


\section{Materials, Synthesis, and Testing Methods}

2.1. Materials. Chemical composition and particle size distribution of PI 42.5 Portland cement, slag, fly ash, and metakaolin are available in Table 1 and Figure 1 . The average particle size of PI 42.5 Portland cement, slag, fly ash, and metakaolin was $36.96 \mu \mathrm{m}, 20.30 \mu \mathrm{m}, 33.69 \mu \mathrm{m}$, and $6.44 \mu \mathrm{m}$, respectively.

Chemical agents include the following: amyl alcohol ethoxylates (TPEG), with a molecular weight of 2400 , analytically pure acrylic acid (AA), thioglycolic acid, toluenesulfonic acid, hydroquinone, benzoyl peroxide, polyethylene glycol-200 (PEG-200), hydrogen peroxide, ascorbic acid (Vc), ammonium persulfate, dibutyl tin dilaurate, and deionized water.

2.2. Synthesis. The structure of the synthesized polycarboxylate is shown in Figure 2. To guarantee double bond molarity in the preparation and to control the concentration of free radical and molecular weight modifier, the molar ratio between acrylic acid and TPEG was adjusted. In synthesis, the solution containing $120 \mathrm{~g}$ of TPEG, $80 \mathrm{ml}$ of pure water, $10 \mathrm{~g}$ of PEG-200 was fully stirred in a $250 \mathrm{ml}$ four-neck round bottom flask. After that, another solution which contained $0.1 \mathrm{~g}$ of dibutyltin dilaurate, $1.2 \mathrm{~g}$ of toluene sulphonic acid, and a certain quantity of $30 \%$ hydrogen peroxide was added. In addition, two groups of solutions (group A and group B) were prepared for the next step.

Group A: certain quantity of acrylic acid, sodium bisulfite, Vc or rongalite, and proper quantity of water.

Group B: certain quantity of thioglycolic acid, ammonium persulfate, and proper quantity of water.

Using a peristaltic pump to $\mathrm{A}$ and $\mathrm{B}$ components, a beaker with $2 \mathrm{ml} / \mathrm{min}$ rate dropped; the process was about $1.5 \mathrm{~h}$. After adding some $\mathrm{NaOH}$ and adjusting the $\mathrm{PH}$ value to 6-7, we obtained three kinds of polycarboxylates called PCE-1, PCE-2, and PCE-3.

\subsection{Testing Methods}

2.3.1. Surface Tension Test. We tested the surface tension of the polycarboxylate sample synthesized by using A-60 type automatic surface tension meter produced by Kono Industries, Ltd. in the USA. Take $50 \mathrm{~g}$ of cementitious material in a beaker with a fixed water-to-binder ratio of 0.4 . Mix the polycarboxylate with $20 \mathrm{~g}$ of water and add it to the beaker. Stir at low speed for $2 \mathrm{~min}$ and then quickly for $1 \mathrm{~min}$. Centrifuge the cement paste and take the supernatant to test its liquid surface tension.

2.3.2. Flowability. According to GB/T8077-2000 "concrete admixture homogeneity test methods," we did hollow cylindrical tryout test its initial flowability, paste flowability of $1 \mathrm{~h}$ and $2 \mathrm{~h}$.

2.3.3. Effect of Polycarboxylate and Supplementary Cementitious Materials Dosage on Hydration Heat Evolution of Cement Pastes. We used the "TAM Air" eight-channel isothermal microcalorimeter produced in Sweden test and recorded the hydration heat and heat release rate of cementitious material paste for quantitative analysis. We took $4 \mathrm{~g}$ cementitious materials in a beaker, wherein replacement of slag, fly ash, and metakaolin was 0 and $10 \%$, solid content of polycarboxylate was $0,0.1 \%, 0.3 \%$, and $0.5 \%$, and the water-binder ratio was set at 0.4 .

2.3.4. Effect of Polycarboxylate and Supplementary Cementitious Materials Dosage on Compressive Strength of Cement Mortar. According to Sections 2.3.1 and 2.3.2, we choose the best polycarboxylate synthesized, prepare cement mortar in the water-binder ratio of 0.3 and sand-cement ratio of $2: 1$, wherein replacement of slag, fly ash, and metakaolin was 0 , $10 \%$ and $20 \%$, solid content of polycarboxylate was $0.8 \%-$ $1.0 \%$, and the flowability of cement mortar was kept at $140 \mathrm{~mm}$ or more, forming a set of $40 \mathrm{~mm} \times 40 \mathrm{~mm} \times 160 \mathrm{~mm}$ of the test specimen. We tested the triple mold, which was cured 24 hours with mold curing oven temperature maintained at $20^{\circ} \mathrm{C} \pm 1^{\circ} \mathrm{C}$ and relative humidity of not less than $90 \%$, and then removed the mold and put them into steam curing chamber wherein temperature was $20^{\circ} \mathrm{C} \pm 3^{\circ} \mathrm{C}$. At last, we measured the strength of cement mortar in 1 day, 3 days, 7 days, 14 days, 28 days, 56 days, and 90 days.

2.3.5. Effect of Polycarboxylate and Supplementary Cementitious Materials Dosage on Autogenous Shrinkage of Cement Mortar. According to Sections 2.3.1 and 2.3.2, we choose the best polycarboxylate and prepared cement mortar in the water-binder ratio of 0.3 and sand-cement ratio of $2: 1$, wherein replacement of slag, fly ash, and metakaolin was 0 , $10 \%$, and $20 \%$, solid content of polycarboxylate was $0.8 \%-$ $1.0 \%$, and the flowability of cement mortar was kept at $140 \mathrm{~mm}$ or more. Then we measured and recorded autogenous shrinkage change from 72 hours after forming cement mortar, using bellows and a noncontact measuring probe.

2.3.6. Effect of Polycarboxylate and Supplementary Cementitious Materials Dosage on Drying Shrinkage of Cement Mortar. According to JC/T603-2004 "cement mortar drying shrinkage test methods," we prepared cement mortar in the water-binder ratio of 0.3 and sand-cement ratio of $2: 1$, wherein replacement of slag, fly ash, and metakaolin was 0 , $10 \%$, and $20 \%$, solid content of polycarboxylate was $0.8 \%-$ $1.0 \%$, and the flowability of cement mortar was kept at $140 \mathrm{~mm}$ or more. Also, we used the best polycarboxylate from Sections 2.3.1 and 2.3.2.

\section{Results and Discussion}

3.1. New Polycarboxylate's Surface Tension and Its Influence on the Flowability of Cement Paste. General polycarboxylate called PCA, synthesized three kinds of polycarboxylate called PCE-1, PCE-2, and PCE-3. Their surface tensions are available in Table 2. Their surface tension was $52.8 \mathrm{mN} / \mathrm{m}$, $46.70 \mathrm{mN} / \mathrm{m}, 42.33 \mathrm{mN} / \mathrm{m}$, and $40.12 \mathrm{mN} / \mathrm{m}$, respectively. Compared with PCA, PCE-3 lowered surface tension by 
Table 1: Chemical composition of PI 42.5 Portland cement, slag, fly ash, and metakaolin (\%).

\begin{tabular}{lccccccccrrr}
\hline \multirow{2}{*}{ Material } & \multicolumn{10}{c}{ Chemical composition (\%) } & \multicolumn{1}{c}{ coss } \\
& $\mathrm{SiO}_{2}$ & $\mathrm{Al}_{2} \mathrm{O}_{3}$ & $\mathrm{Fe}_{2} \mathrm{O}_{3}$ & $\mathrm{CaO}$ & $\mathrm{MgO}$ & $\mathrm{SO}_{3}$ & $\mathrm{TiO}_{2}$ & $\mathrm{~K}_{2} \mathrm{O}$ & $\mathrm{Na}_{2} \mathrm{O}$ & Total & Loss \\
\hline Cement & 21.18 & 4.73 & 3.41 & 62.49 & 2.53 & 2.83 & - & - & 0.56 & 97.73 & 1.76 \\
Slag & 33 & 13.91 & 0.82 & 39.11 & 10.04 & - & 0.10 & 1.91 & - & 98.89 & 1.12 \\
Fly ash & 54.29 & 22.55 & 8.46 & 5.58 & 2.56 & 0.53 & - & 1.80 & 0.67 & 96.44 & 2.37 \\
Metakaolin & 51.40 & 36.17 & 0.51 & 0.05 & 0.64 & - & 0.13 & 0.15 & 0.13 & 89.18 & 10.37 \\
\hline
\end{tabular}

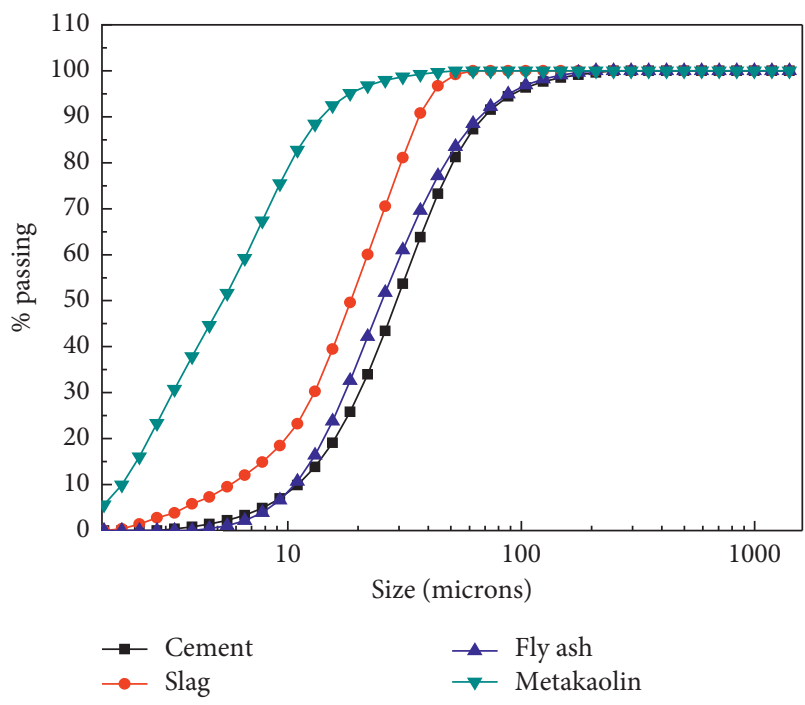

FIGURE 1: Size distribution of cement, slag, fly ash, and metakaolin.

24.0\%. Obviously, with the molar ratio of AA with TPEG increases, the surface tension was lowered; however, its viscosity and molecular weight were increased.

From Tables 2 and 3, we could see some differences in cement paste flowability by using polycarboxylate synthesized in a different molar ratio of AA and TPEG. Since the acid-ether ratio in the synthesis of PCE-1, PCE-2, and PCE-3 was $3: 1,6: 1$, and $9: 1$; meanwhile the ratio of acid-ether in PCA was $1: 1$, the adsorption points in their structures of PCE-1, PCE-2, and PCE-3 were much more than that of PCA. So, PCE-1, PCE-2, and PCE-3 have better surface tension and flowability than PCA [5]. In addition, their initial value changed a little, but the difference had become more and more obvious as time went by. From these two points, surface tension and paste flowability, we knew that we could synthesis better polycarboxylate by increasing the molar ratio of AA and TPEG to $6: 1$ or more.

\subsection{Effect of SCMs on Hydration Heat Evolution of Cement} Pastes Containing PCE-2. Figures 3-10 show the effect of PCE-2 and supplementary cementitious materials on hydration heat evolution of cement mortar, wherein replacement of slag, fly ash, and metakaolin was 0 and $10 \%$, solid content of polycarboxylate was $0,0.1 \%, 0.3 \%$, and $0.5 \%$.

It could be seen that the heat release rate and total heat for the process of cement hydration had been slowed down after incorporation of polycarboxylates, and the greater the dosage had been used, the more obvious the effects would be

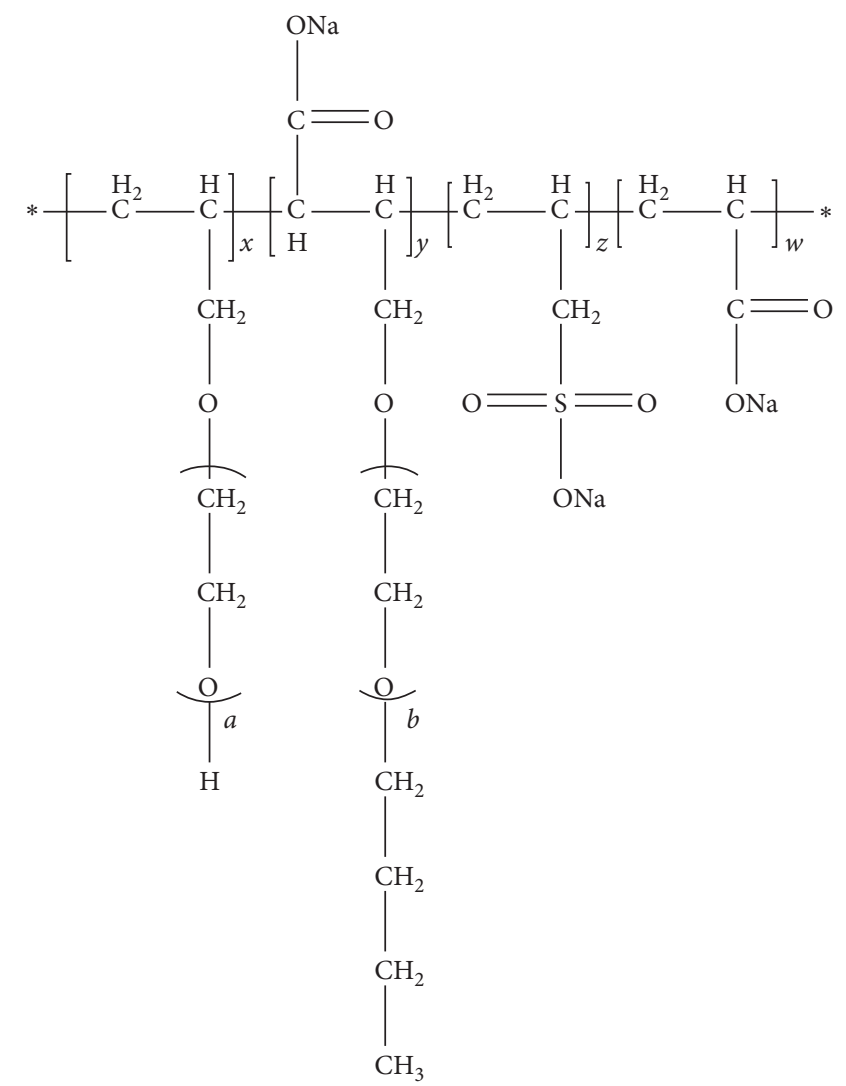

FIGURE 2: Structure of synthesized polycarboxylate.

TABLE 2: Surface tension of PCE-1, PCE-2, and PCE-3.

\begin{tabular}{lccc}
\hline Sample & $\begin{array}{c}n(\mathrm{AA}) / n \\
(\mathrm{TPEG})\end{array}$ & $\begin{array}{c}\text { Solid content } \\
(\%)\end{array}$ & $\begin{array}{c}\text { Surface } \\
\text { tension }\left(\mathrm{mN} \cdot \mathrm{m}^{-1}\right)\end{array}$ \\
\hline Water & & - & 72.9 \\
PCA & $1: 1$ & 25 & 52.8 \\
PCE-1 & $3: 1$ & 25 & 46.40 \\
PCE-2 & $6: 1$ & 25 & 42.33 \\
PCE-3 & $9: 1$ & 25 & 40.12 \\
\hline
\end{tabular}

TAble 3: Paste flowability of PCE-1, PCE-2, and PCE-3.

\begin{tabular}{lcccc}
\hline \multirow{2}{*}{ Sample } & Dosage (\%) & \multicolumn{3}{c}{ Flowability $(\mathrm{mm})$} \\
& & Initial & $1 \mathrm{~h}$ & $2 \mathrm{~h}$ \\
\hline PCE-1 & 0.15 & 280 & 190 & 135 \\
PCE-2 & 0.15 & 315 & 297 & 287 \\
PCE-3 & 0.15 & 305 & 290 & 275 \\
\hline
\end{tabular}




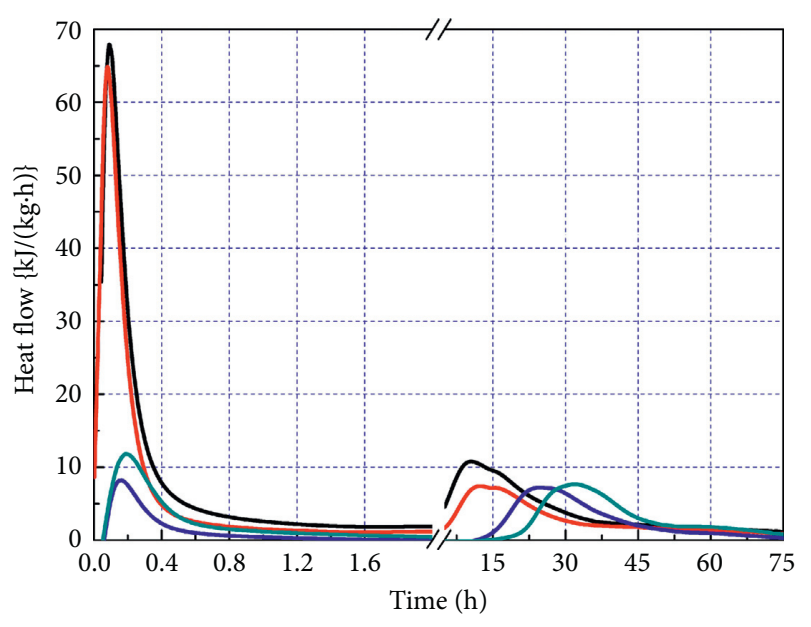

$\begin{array}{ll}-0 \% \text { polycarboxylate } & -0.3 \% \text { polycarboxylate } \\ -0.1 \% \text { polycarboxylate } \quad-0.5 \% \text { polycarboxylate }\end{array}$

Figure 3: Effect of PCE-2 dosage on the hydration heat flow of cement pastes.

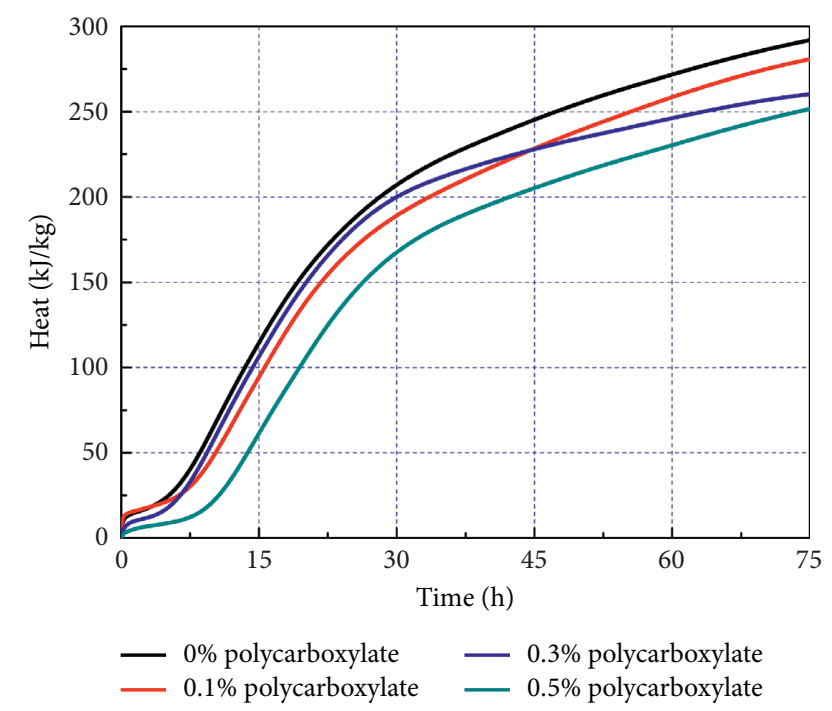

Figure 4: Effect of PCE-2 dosage on hydration heat of cement pastes.

$[6,7]$. After the incorporation of polycarboxylates, it would be adsorbed on the surface of cement particles at the beginning of cement hydration. In addition, active groups in molecular chains of polycarboxylate (such as - $\mathrm{COO}-、-\mathrm{SO}_{3^{-}}$) and hydration ions (such as $\mathrm{Ca}^{2+}$ ) would combine together and generate unstable complexes in alkaline medium of cement hydration. In this way, cement hydration would be delayed and initial precipitation and growth phase of hydration products would be inhibited. Moreover, the effect was enhanced with increasing dosage of polycarboxylate $[8,9]$. In other words, dissolve peak in hydration would be declined as shown in Figures 3 to 10. Furthermore, because the side chain in polycarboxylate with adsorbs, complexes, retards, and other effects on Portland cement, it inhibited the hydration of hydrated minerals and the growth of hydration

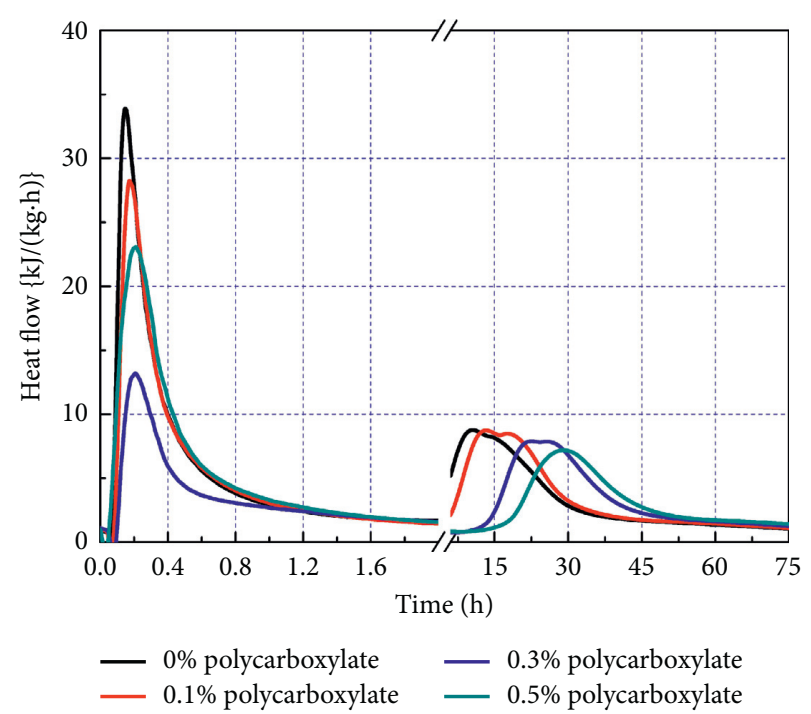

FIgURE 5: Effect of PCE-2 dosage on hydration heat flow of cement pastes with $10 \%$ slag.

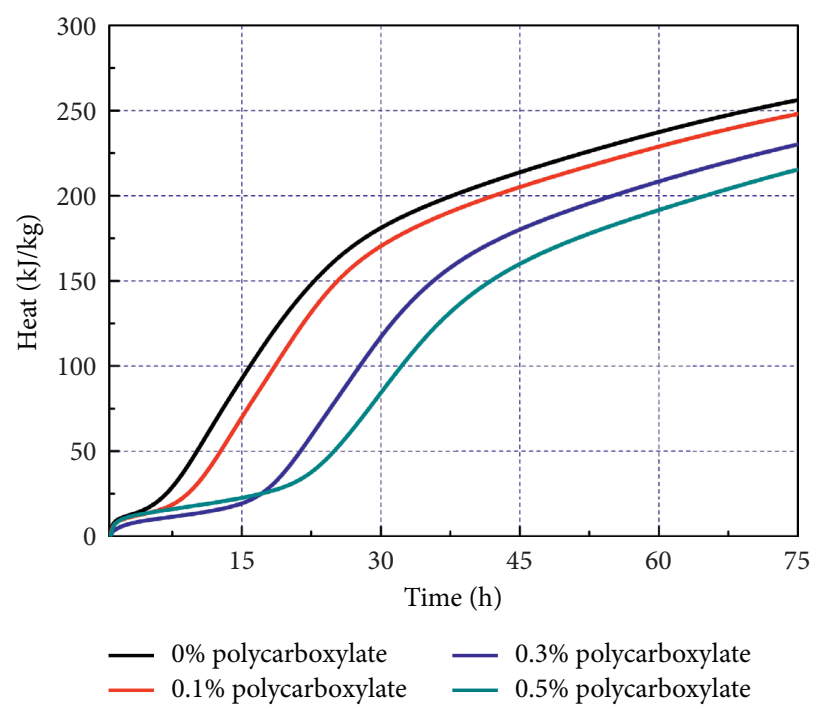

FIGURE 6: Effect of PCE-2 dosage on hydration heat of cement pastes with $10 \%$ slag.

products and cost more time to overcome the hydration energy barrier. So, the induction period of hydration increased and the second exothermic peak was delayed and weakened. Similarly, as the amount of polycarboxylic acid increased, the effect of this delay was more obvious and the second exothermic peak shifted to the right side [10].

We also found that the incorporation of supplementary cementitious materials could reduce the hydration heat release rate and total release heat of cement pastes under the same experimental conditions. Free lime, gypsum, and aluminate in supplementary cementitious materials, which were quickly dissolved in cement hydration, would promote hydration on surface of cement and lead to a rapid heat release system [11]. However, further action between water 


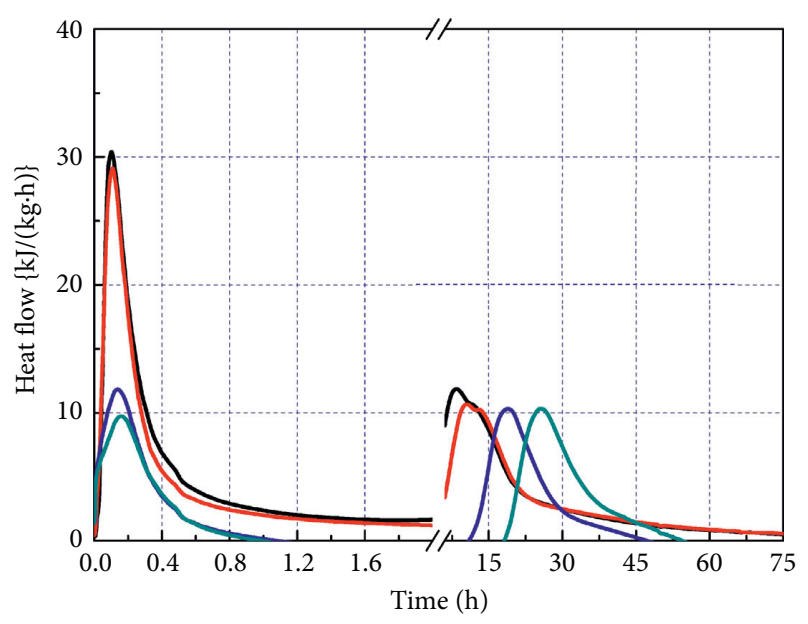

$\begin{array}{ll}-0 \% \text { polycarboxylate } & -0.3 \% \text { polycarboxylate } \\ -0.1 \% \text { polycarboxylate } & -0.5 \% \text { polycarboxylate }\end{array}$

FIGURE 7: Effect of PCE-2 dosage on hydration heat flow of cement pastes with $10 \%$ fly ash.

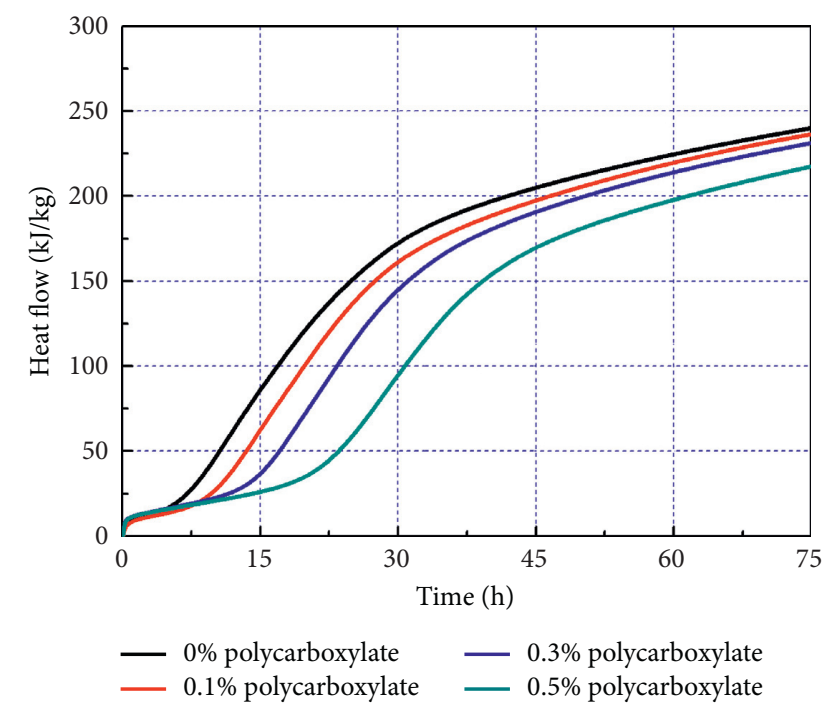

FIGURE 8: Effect of PCE-2 dosage on hydration heat of cement pastes with $10 \%$ fly ash.

and cement would be hindered because the particles of cement were coated by sulphoaluminate and calcium silicate produced in early times of hydration [12]. After that, the heat release rate began to rise again, reaching the highest value (the second peak). The reason was considered that the cladding layer under osmotic and crystallized pressure continued to be destroyed, and the reaction between water and cement was accelerated [13]. Due to the replacement of supplementary cementitious materials in cement, the content of cement was lowered that made the total heat release reduced [14]. In the second and the third phases in hydration, named induced and accelerated, respectively, due to the dilution of cement, the total heat release would be reduced with increasing the dosage of supplementary cementitious materials [15].
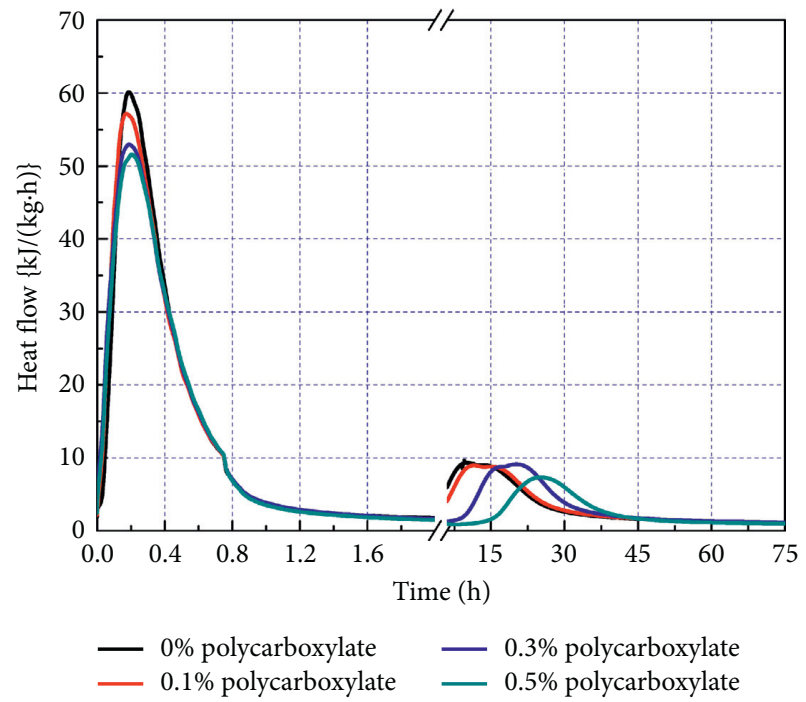

FIgURE 9: Effect of PCE-2 dosage on hydration heat flow of cement pastes with $10 \%$ metakaolin.

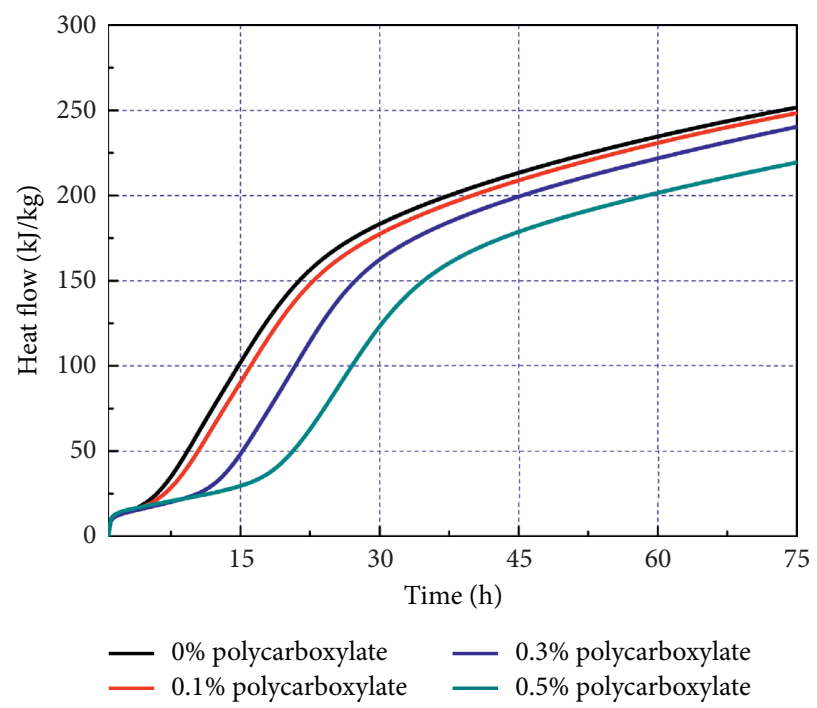

FIGURE 10: Effect of PCE-2 dosage on hydration heat of cement pastes with $10 \%$ metakaolin.

\subsection{Effect of SCMs on Compressive Strength of Cement Mortar} Containing PCE-2. PCE-2 was chosen to prepare cement mortar in the water-binder ratio of 0.3 and the sand-cement ratio of $2: 1$. Then, we measured the compressive strength of cement mortar in different ages.

Figures 11-13 show the effect of PCE-2 and supplementary cementitious materials on compressive strength of cement mortar in different ages. We could see that the largest increases of slag, fly ash, and metakaolin were $24.5 \%, 23.1 \%$, and $24.3 \%$, respectively. Because these three supplementary cementitious materials had a smaller particle size than cement, whose pores could be better filled, thus particle packing system became more close [16]. Moreover, supplementary cementitious materials had good shape effects, 


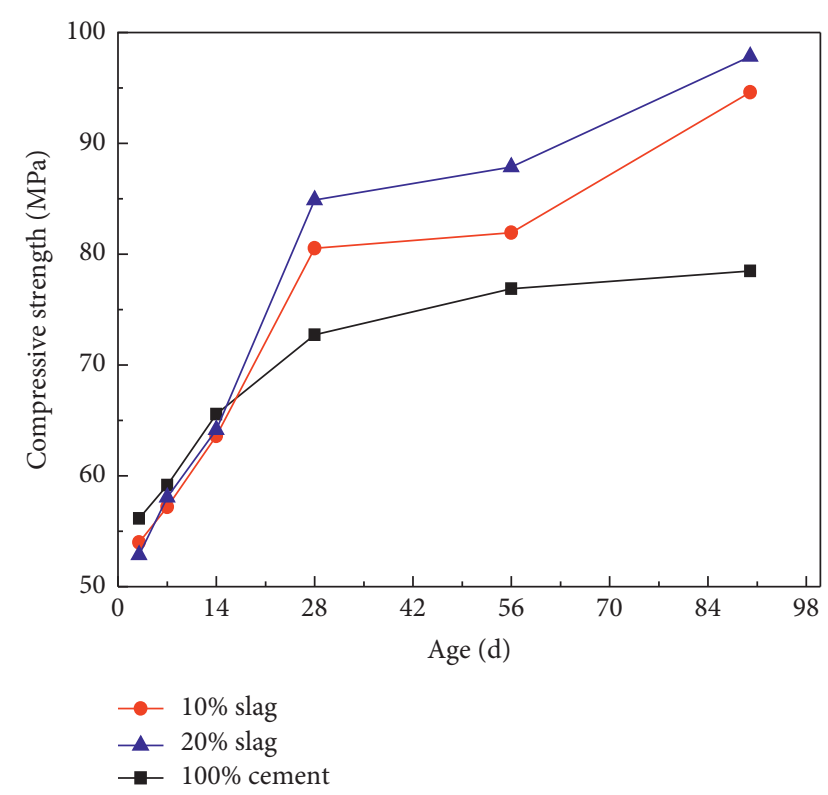

FIGURE 11: Effect of PCE-2 and slag dosage on the compressive strength of cement mortar.

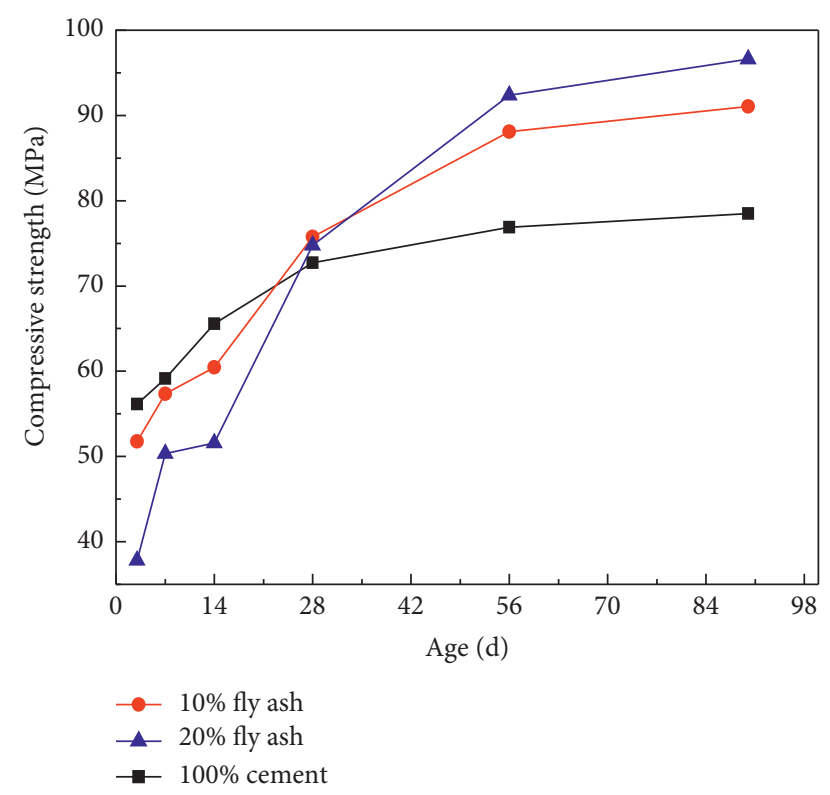

FIGURE 12: Effect of PCE-2 and fly ash dosage on compressive strength of cement mortar.

good mobility, and smaller adsorption of water, so they could improve the workability of the concrete mixture, which showed some water-reducing effect [17]. In addition, we also found that cement mortar with slag and fly ash dosage showed lower early strength, while the ones with metakaolin dosage showed higher early strength. This was due to the lower pozzolanic activity of slag and fly ash, which required some conditions to have a reaction with cement after seven days usually. At the beginning, supplementary cementitious materials just filled in the hydration products of cement. Until $\mathrm{OH}^{-}$went on eroding supplementary

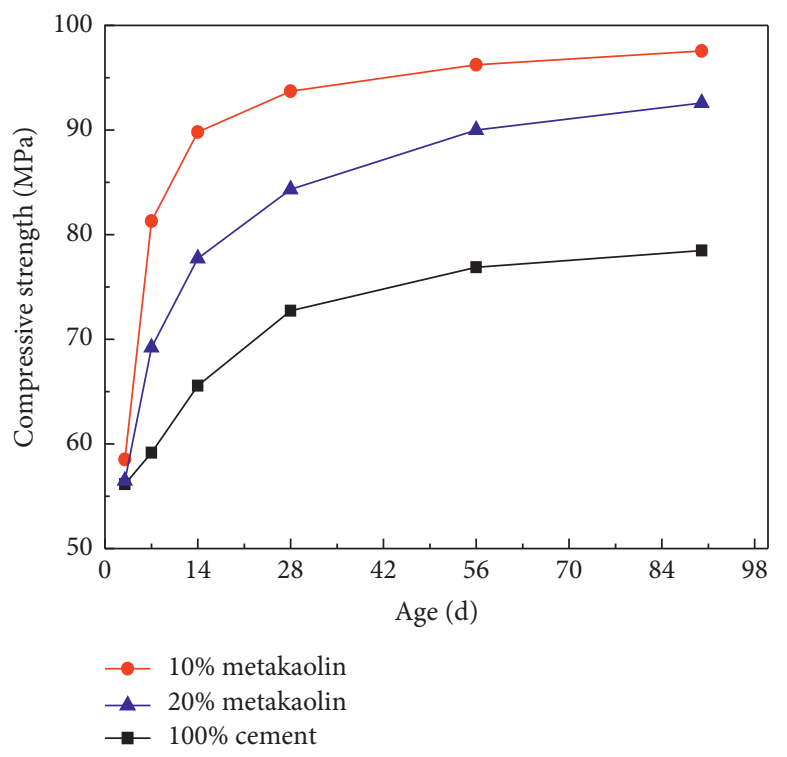

FIGURE 13: Effect of PCE-2 and fly ash dosage on compressive strength of cement mortar.

cementitious materials, they did not react at all. Thus, the strength of cement mortar with slag and fly ash was relatively lower [18]. As to metakaolin, this supplementary cementitious material was made in $600-800^{\circ} \mathrm{C}$, its original structure of $\mathrm{OH}^{-}$and morphology were decomposed, destroyed, and broken down into pieces and poor crystallinity of $\mathrm{Al}_{2} \mathrm{O}_{3} \bullet \mathrm{SiO}_{2}$ relatively; the change in structure made metakaolin have higher pozzolanic activity $[17,18]$. So, in early times of hydration reaction of cement, $\mathrm{Ca}^{2+}, \mathrm{Al}^{3+}, \mathrm{AlO}_{4}{ }^{5+}$, and $\mathrm{SiO}_{4}{ }^{4-}$ in metakaolin would go into the solution, generating new hydration products and improving the early strength of cement mortar. The functional groups in the side chain bonded with the calcium ions in the mortar, which could produce the corresponding ionic cross-linked structure and lead to a significant increase in the degree of polymerization of the hydration product C-S-H [19]. Furthermore, the introduction of tricarboxylic groups and sulphonic acid groups in the molecular structure made the process of transforming the hydrated gel into crystal become slower. It was beneficial to form more complete crystals and improve the density of crystals, resulting in higher strength mortar [20].

\subsection{Effect of SCMs on Autogenous Shrinkage of Cement Mortar} Containing PCE-2. Figures 14-16 show the effect of PCE-2 and supplementary cementitious materials on drying shrinkage of cement mortar in different ages. Some results from the figures could show that the incorporation of polycarboxylates and slag, fly ash, and metakaolin slowed down the shrinkage of cement to some extent, and the influence descending order was fly ash $>$ metakaolin $>$ slag.

From Figures 14-16, it could be seen that autogenous shrinkage was finished in less than $12 \mathrm{~h}$ substantially in hydration. Under the same conditions, adding supplementary cementitious materials into cement mortar could 


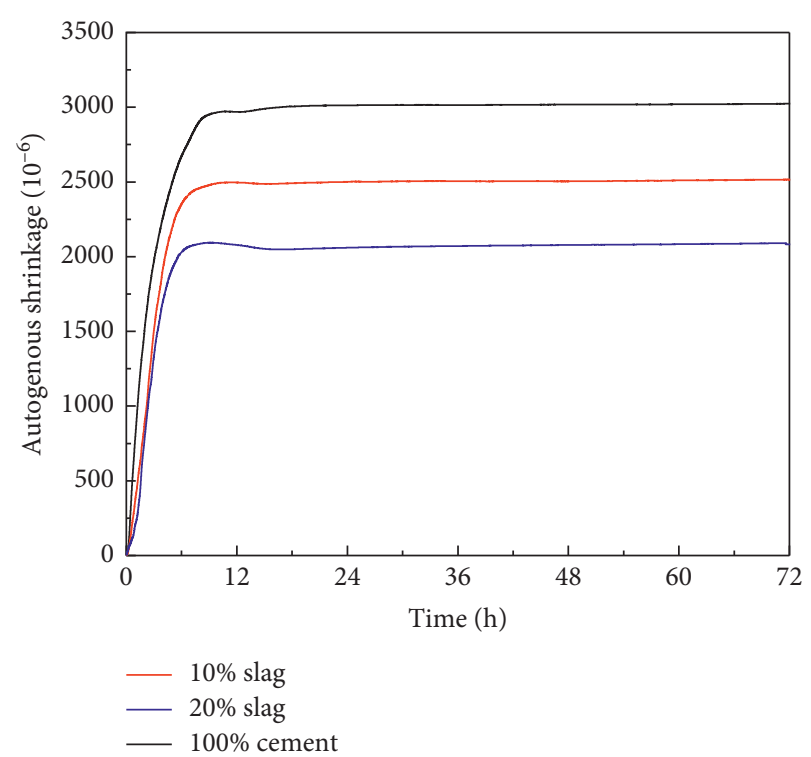

FIgUre 14: Effect of PCE-2 and slag dosage on autogenous shrinkage of cement mortar.

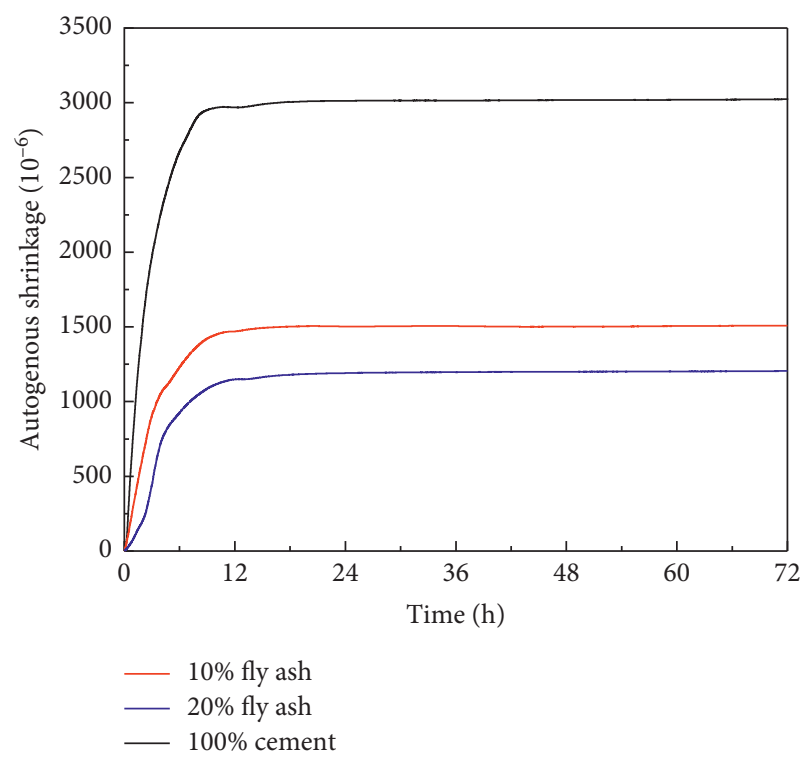

Figure 15: Effect of PCE-2 and fly ash dosage on autogenous shrinkage of cement mortar.

reduce its autogenous shrinkage effectively. The amount of reduction of slag, fly ash, and metakaolin was $31.1 \%, 60.1 \%$, and $57.4 \%$, respectively. Apparently, the dosage of slag, fly ash, and metakaolin could effectively reduce autogenous shrinkage of cement mortar.

After being mixed with cement, slag, whose activity was low, would replace some parts of cement, which was equivalent to increasing water-binder ratio. Thus, it could improve the change of autogenous shrinkage caused by the drying effect. In other words, autogenous shrinkage was reduced and its effect increased when the content of slag was

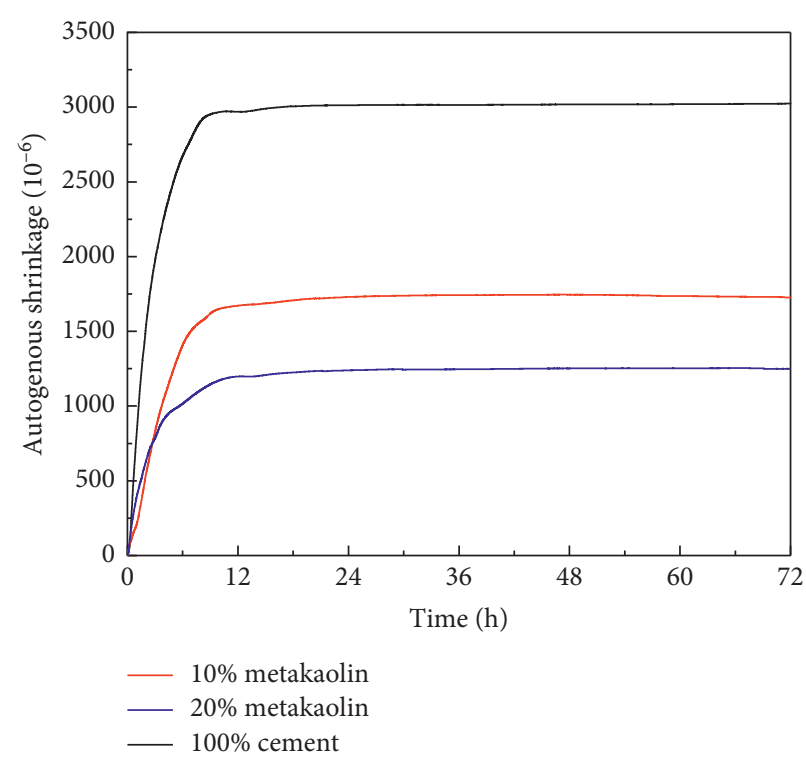

Figure 16: Effect of PCE-2 and metakaolin dosage on autogenous shrinkage of cement mortar.

becoming larger. The speed and extent of participation of fly ash, which had slow pozzolanic activity in the hydration reaction relatively, was much lower than cement. In early times, most products of hydration came from cement only. Small products of hydration meant small amount of chemical shrinkage [21]. In addition, particles of fly ash could inhibit chemical shrinkage [22]. Above those, autogenous shrinkage of cement mortar with fly ash was decreased. Although metakaolin had reactive pozzolanic activity relatively, which could make many hydration products of cement in early times, on the other hand, this process could lead to intense heat as well. So, autogenous shrinkage would be reduced instead, due to the thermal expansion of hydration products [23]. In addition, after the metakaolin was calcined at high temperature, the pore size between the particles increased. This unique interlayer structure allowed metakaolin to adsorb more polycarboxylate molecules, resulting in a reduction in the amount of free water in the cement mortar mixed with metakaolin. The autogenous shrinkage of mortar would decrease sharply with the increase of metakaolin content [24].

3.5. Effect of SCMs on Drying Shrinkage of Cement Mortar Containing PCE-2. PCE-2 was chosen to prepare cement mortar in the water-binder ratio of 0.3 and sand-cement ratio of $2: 1$. Then we measured drying shrinkage of cement mortar in different ages.

As it could be seen from Figures 17-19, adding supplementary cementitious materials into cement mortar could reduce its drying shrinkage effectively. The amount of reduction of slag, fly ash, and metakaolin was $8.8 \%, 21.2 \%$, and $21.9 \%$, respectively. But the reason why these three kinds of supplementary cementitious materials could achieve was different slightly. 


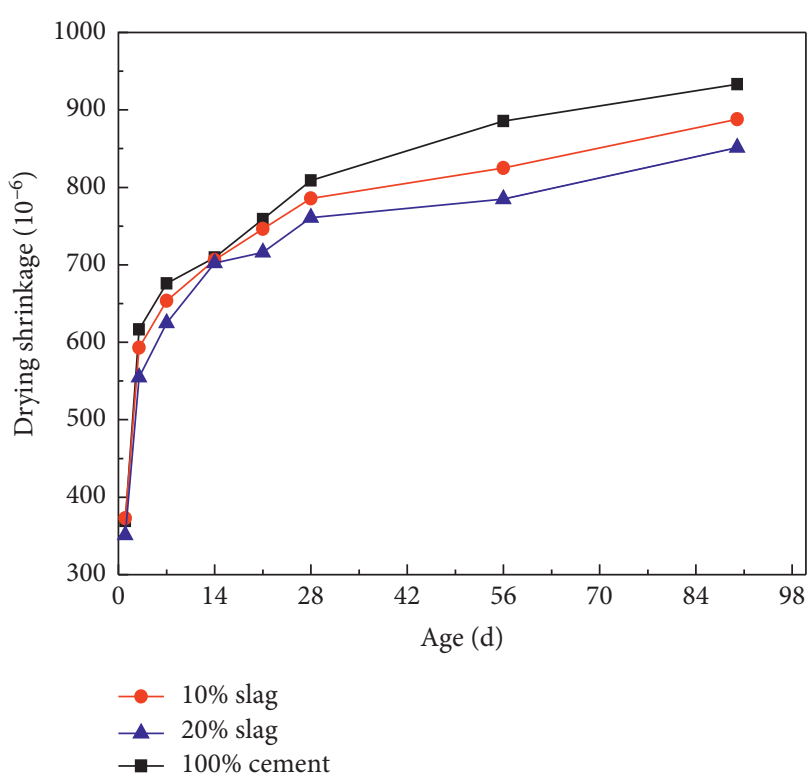

FIGURE 17: Effect of PCE-2 and slag dosage on drying shrinkage of cement mortar.

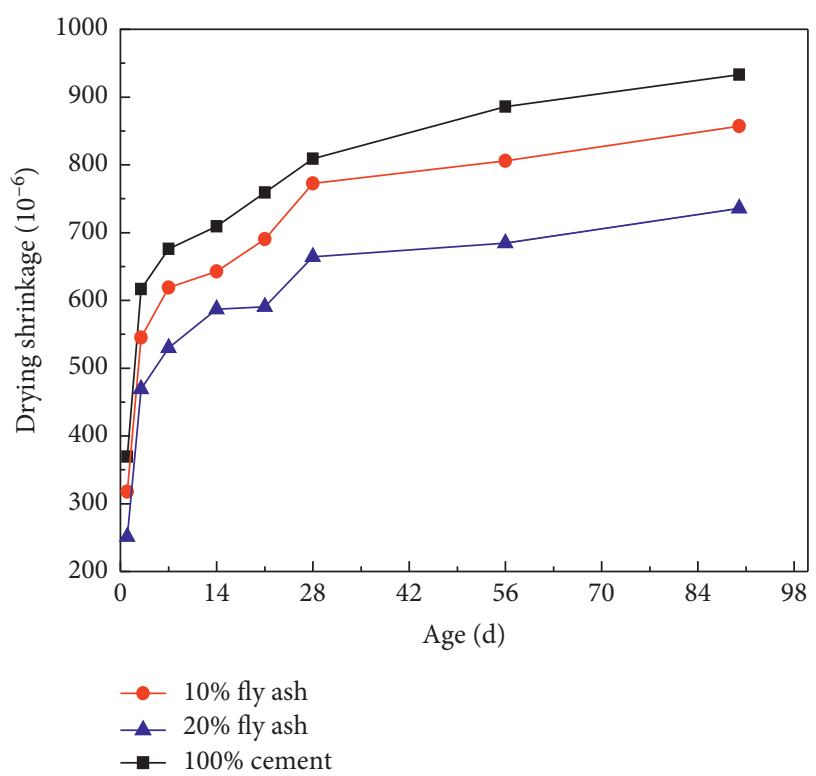

Figure 18: Effect of PCE-2 and fly ash dosage on drying shrinkage of cement mortar.

Being similar to the particle shape of cement, slag had irregular particles, small fineness, vitreous, low degree of crystallinity, particle disordered arrangement, and large specific surface area. After incorporation, it could refine the pore structure of paste, reduce the pore connectivity, and increase the difficulty of moisture migration under dry conditions, which had a positive effect on drying shrinkage, and thus drying shrinkage was decreased.

Fly ash had spherical particles, strong adsorption, fluffy structures, and microporous. The speed and extent of involvement of fly ash in cement hydration reaction were much lower than its pozzolanic activity $[25,26]$. It needed to stimulate

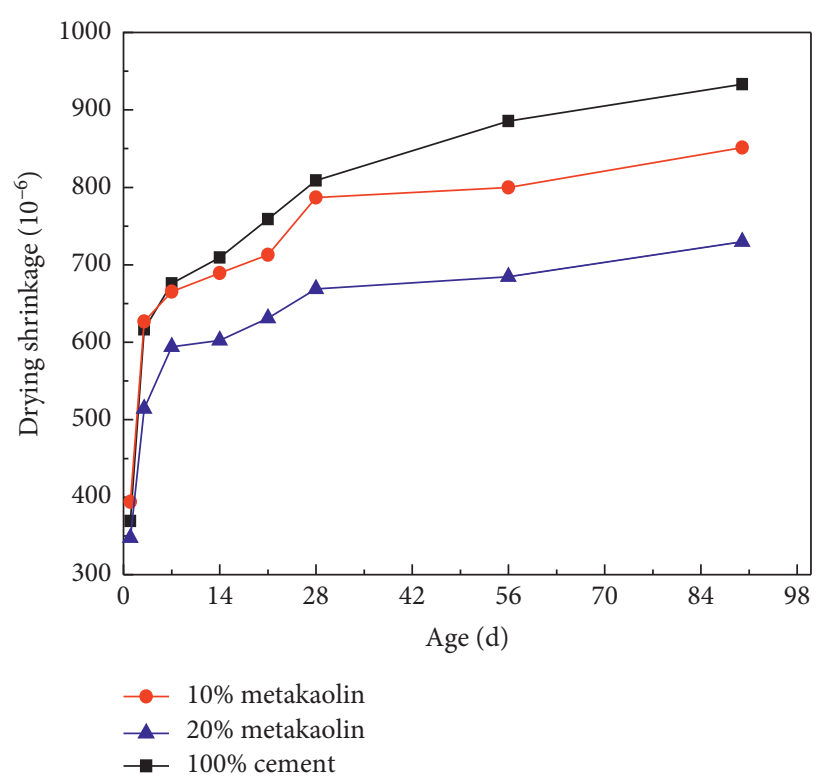

Figure 19: Effect of PCE-2 and metakaolin dosage on drying shrinkage of cement mortar.

with cement hydration products in the early times of hydration. Thus the inhibition of drying shrinkage with fly ash on the hydration system was very obvious. Although with the increase of fly ash, effective water-binder ratio cement mortar increased, at the same time, the elastic modulus of fly ash particles was also higher than the cement's, which played the role of limiting the slurry shrinkage in cement mortar. All those above were so effective in reducing drying shrinkage in cement mortar with fly ash $[27,28]$.

After being calcined at high temperature, the original structure of $\mathrm{OH}$ - and morphology in metakaolin was decomposed, destroyed, and broken down into pieces and poor crystallinity of $\mathrm{Al}_{2} \mathrm{O}_{3} \bullet \mathrm{SiO}_{2}$ relatively, whose change in structure of metakaolin made this kind of higher pozzolanic activity [29]. With the dosage of metakaolin, its pozzolanic and aggregate activity could effectively reduce content and evaporation of free water in the interspace of cement mortar, making migration become difficult to moisture, thus reducing drying shrinkage development at last $[30,31]$.

\section{Conclusion}

In this article, the influence of some factors on the synthesis of polycarboxylate at room temperature was discussed. The best product was selected to study its effects on the hydration heat evolution, compressive strength, autogenous shrinkage, and drying shrinkage of cement pastes with different kinds and contents of supplementary cementitious materials. The following conclusions could be obtained based on the above experimental results and discussions:

(1) From the experimental results, appropriately increasing the molar ratio of AA with TPEG contributed to the surface tension of polycarboxylates, cement paste flowability, and the optimum mixing ratio was $6: 1$. 
(2) After incorporation of polycarboxylates, heat flow and total heat for the process of cement hydration had been slowed down. The greater the dosage used, the more obvious the effects would be. The dosage of supplementary cementitious materials could reduce hydration heat release rate and the total release heat of cement pastes.

(3) The compressive strength of cement mortar after the age of 90 days was greatly contributed by supplementary cementitious materials. Meanwhile, the largest increases of slag, fly ash, and metakaolin were $24.5 \%, 23.1 \%$, and $24.3 \%$, respectively.

(4) The incorporation of polycarboxylates and slag, fly ash, metakaolin slowed down the autogenous shrinkage of cement mortar, and the influence descending order was fly ash $>$ metakaolin $>$ slag.

(5) Adding supplementary cementitious materials into cement mortar could reduce its drying shrinkage. The largest decrease of slag, fly ash, and metakaolin was $8.8 \%, 21.2 \%$, and $21.9 \%$.

\section{Data Availability}

The processed data required to reproduce these findings cannot be shared at this time as the data also forms part of an ongoing study.

\section{Conflicts of Interest}

The authors declare that they have no conflicts of interest.

\section{Authors' Contributions}

Shuncheng Xiang contributed to conceptualization, methodology, investigation, data curation, formal analysis, and writing the original draft. Yingli Gao contributed to project administration, funding acquisition, and supervision. Caijun Shi revised the manuscript.

\section{Acknowledgments}

This project was supported by the National Key R\&D Program of China (Grant number: 2018YFB1600100) and Open Fund (Grant number: kfj190503) of Key Laboratory of Special Environment Road Engineering of Hunan Province (Changsha University of Science and Technology).

\section{References}

[1] I. J. D. Vries and R. B. Polder, "Hydrophobic treatment of concrete," Construction and Building Materials, vol. 11, no. 4, pp. 259-265, 1997.

[2] B. He, Y. Gao, L. Qu, K. Duan, W. Zhou, and G. Pei, "Characteristics analysis of self-luminescent cement-based composite materials with self-cleaning effect," Journal of Cleaner Production, vol. 225, pp. 1169-1183, 2019.

[3] Y. Gao, L. Qu, B. He, K. Dai, Z. Fang, and R. Zhu, "Study on effectiveness of anti-icing and deicing performance of superhydrophobic asphalt concrete," Construction and Building Materials, vol. 191, pp. 270-280, 2018.
[4] K. R. Duan, Y. L. Gao, H. Yao et al., "Comparison of performances of early aged pre-vibrated cement-stabilized macadam formed by different compactions," Construction and Building Materials, vol. 239, 2020.

[5] K. Moradllo, M. Shekarchi, and M. Hoseini, “Time-dependent performance of concrete surface coatings in tidal zone of marine environment," Construction and Building Materials, vol. 30, pp. 198-205, 2012.

[6] B. Pigino, A. Leemann, E. Franzoni, and P. Lura, "Ethyl silicate for surface treatment of concrete - Part II: characteristics and performance," Cement and Concrete Composites, vol. 34, no. 3, pp. 313-321, 2012.

[7] Y. Dang, N. Xie, A. Kessel, E. McVey, A. Pace, and X. Shi, "Accelerated laboratory evaluation of surface treatments for protecting concrete bridge decks from salt scaling," Construction and Building Materials, vol. 55, no. 0, pp. 128-135, 2014.

[8] H. Li, Z. Yi, and Y. Xie, "Progress of silane impregnating surface treatment technology of concrete structure," Materials Review, vol. 26, no. 3, pp. 120-125, 2012.

[9] M. Medeiros and P. Helene, "Efficacy of surface hydrophobic agents in reducing water and chloride ion penetration in concrete," Materials and Structures, vol. 41, no. 1, pp. 59-71, 2008.

[10] M. H. F. Medeiros and P. Helene, "Surface treatment of reinforced concrete in marine environment: influence on chloride diffusion coefficient and capillary water absorption," Construction and Building Materials, vol. 23, no. 3, pp. 1476-1484, 2009.

[11] M. Jones, R. Dhir, and J. Gill, "Concrete surface treatment: effect of exposure temperature on chloride diffusion resistance," Cement and Concrete Research, vol. 25, no. 1, pp. 197-208, 1995.

[12] J. Zhang, L. Ding, F. Li, and J. Peng, "Recycled aggregates from construction and demolition wastes as alternative filling materials for highway subgrades in China," Journal of Cleaner Production, vol. 255, p. 120223, 2020.

[13] J. L. Thompson, M. R. Silsbee, P. M. Gill, and B. E. Scheetz, "Characterization of silicate sealers on concrete," Cement and Concrete Research, vol. 27, no. 10, pp. 1561-1567, 1997.

[14] D. A. Kagi and K. B. Ren, "Reduction of water absorption in silicate treated concrete by post-treatment with cationic surfactants," Building and Environment, vol. 30, no. 2, pp. 237-243, 1995.

[15] E. Franzoni, H. Varum, M. E. Natali et al., "Improvement of historic reinforced concrete/mortars by impregnation and electrochemical methods," Cement and Concrete Composites, vol. 49, pp. 50-58, 2014.

[16] J. Zhang and F. Gu, "Use of building-related construction and demolition wastes in highway embankment: laboratory and field evaluations," Journal of Cleaner Production, vol. 230, pp. 1051-1060, 2019.

[17] E. Zhang, B. Pigino, and C. Pistolesi, "Ethyl silicate for surface protection of concrete: performance in comparison with other inorganic surface treatments," Cement and Concrete Composites, vol. 44, pp. 69-76, 2013.

[18] L. Jia, Effects of Concrete Surface Treatments on Permeability of the Surface Layer of Concrete, Hunan University, Changsha, China, 2013.

[19] J. Visser, "Influence of the carbon dioxide concentration on the resistance to carbonation of concrete," Construction and Building Materials, vol. 67, pp. 8-13, 2014.

[20] G. W. Groves, A. Brough, I. G. Richardson, and C. M. Dobson, "Progressive changes in the structure of hardened C3S cement 
pastes due to carbonation," Journal of the American Ceramic Society, vol. 74, no. 11, pp. 2891-2896, 1991.

[21] J. He and C. Yang, "Analysis of carbonation of Portland cement concrete," Bulletin of The Chinese Ceramic Society, vol. 28, no. 6, pp. 1225-1229, 2009.

[22] Z. Zhang, H. Wang, and J. L. Provis, "Quantitative study of the reactivity of fly ash in geopolymerization by FTIR," Journal of Sustainable Cement-Based Materials, vol. 1, no. 4, pp. 154-166, 2012.

[23] L. G. Li, J. J. Feng, J. Zhu, S. H. Chu, and A. K. H. Kwan, "Pervious concrete: effects of porosity on permeability and strength," Magazine of Concrete Research, pp. 1-35, 2019.

[24] L. G. Li, Z. Y. Zhuo, J. Zhu, and A. K. H. Kwan, "Adding ceramic polishing waste as paste substitute to improve sulphate and shrinkage resistances of mortar," Powder Technology, vol. 362, pp. 149-156, 2020.

[25] G. Wu, Fourier Transform Infrared Spectroscopy Technology and its Application, Scientific and technological literature press, Oxford, UK, 1994.

[26] G. Li, G. Xiong, Y. lü, and Y. Yin, "The physical and chemical effects of long-term sulphuric acid exposure on hybrid modified cement mortar," Cement and Concrete Composites, vol. 31, no. 5, pp. 325-330, 2009.

[27] X. Zhao, Synthesis of Calcium Silicate Hydrate and its Composition, Structure and Morphology, Wuhan University of Technology, Wuhan, China, 2010.

[28] R. Ylmén, U. Jäglid, B.-M. Steenari, and I. Panas, "Early hydration and setting of Portland cement monitored by IR, SEM and Vicat techniques," Cement and Concrete Research, vol. 39, no. 5, pp. 433-439, 2009.

[29] P. Yu, "Structure of calcium silicate hydrate (C-S-H): near, Mid, and Far infrared spectroscopy," Journal of the American Ceramic Society, vol. 82, no. 3, pp. 742-748, 1999.

[30] R. Ylmén, L. Wadsö, and I. Panas, "Insights into early hydration of Portland limestone cement from infrared spectroscopy and isothermal calorimetry," Cement and Concrete Research, vol. 40, no. 10, pp. 1541-1546, 2010.

[31] Y. Wu, G. Jiang, J. You, and H. Chen, "Progress of research on microstructure amorphous silica," Journal of the Chinese Ceramic Society, vol. 32, no. 1, pp. 57-62, 2004. 